\title{
The Construction of Jacobi and Periodic Jacobi Matrices With Prescribed Spectra
}

\author{
By Warren E. Ferguson, Jr.*
}

\begin{abstract}
The spectral properties of Jacobi and periodic Jacobi matrices are analyzed and algorithms for the construction of Jacobi and periodic Jacobi matrices with prescribed spectra are presented. Numerical evidence demonstrates that these algorithms are of practical utility. These algorithms have been used in studies of the periodic Toda lattice, and might also be used in studies of inverse eigenvalue problems for Sturm-Liouville equations and Hill's equation.
\end{abstract}

1. Introduction. A periodic Jacobi matrix is any real, symmetric matrix of the form

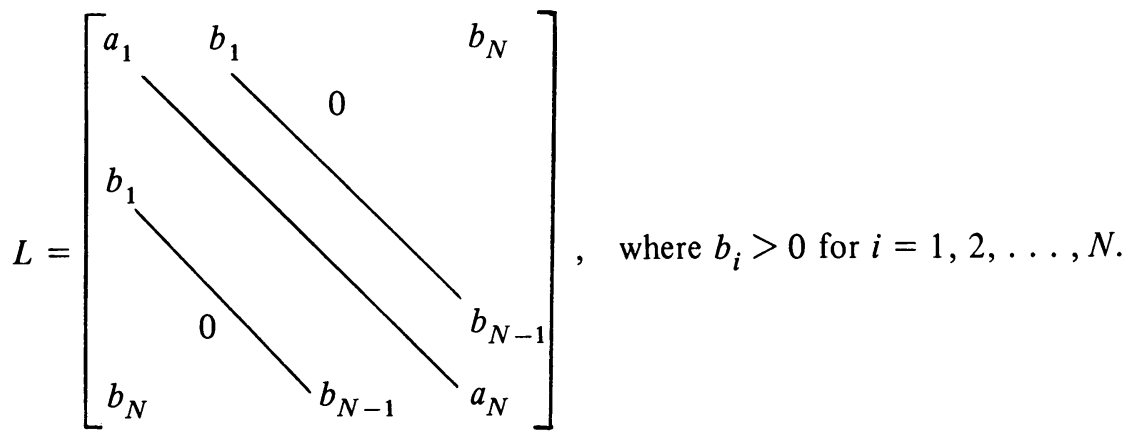

This paper shows how one can characterize the family of periodic Jacobi matrices with prescribed spectra and numerically construct each of its members. For example, there is a nonempty family of periodic Jacobi matrices with $\lambda_{1}, \lambda_{2}, \ldots, \lambda_{N}$ as their eigenvalues if and only if the numbers $\lambda_{1}, \lambda_{2}, \ldots, \lambda_{N}$ are real and can be rearranged so that

$$
\lambda_{1}>\lambda_{2} \geqslant \lambda_{3}>\lambda_{4} \geqslant \lambda_{5}>\ldots .
$$

This family of periodic Jacobi matrices was partially characterized by van Moerbeke [15], and an algorithm which constructs some members of this family was proposed by Boley and Golub [3] .

The results presented in this paper are based upon an analysis of the spectral properties of periodic Jacobi matrices. The derivation of the spectral properties of periodic Jacobi matrices depends quite heavily on the discrete version of Floquet theory,

Received April 6, 1979; revised January 11, 1980.

1980 Mathematics Subject Classification. Primary 15A18, 65F15, 65F30, 65 L15.

* Sponsored by the United States Army under Contract No. DAAG29-75-C-0024. This material is based upon work supported by the National Science Foundation under Grant no. MCS7809525. 
as presented by Flaschka [6], Flaschka and McLaughlin [7], and van Moerbeke [15] .

We develop this version of Floquet theory by using our knowledge of the spectral properties of Jacobi matrices. Recall that a Jacobi matrix is any real, symmetric tridiagonal matrix whose next to diagonal entries are positive. Our canonical Jacobi matrix will be the matrix

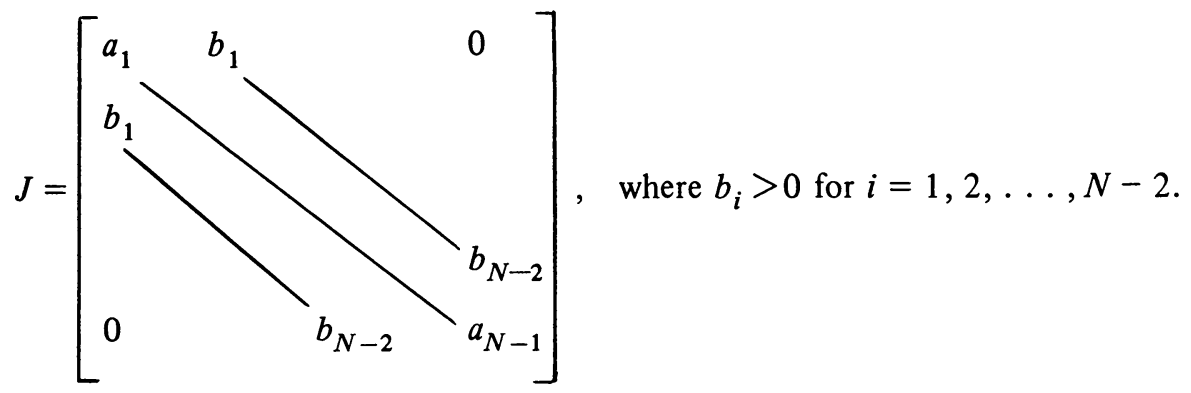

obtained from $L$ by deleting the last row and column.

An algorithm which constructs a Jacobi matrix with prescribed spectra is presented in Section 2. We hasten to point out that essentially the same algorithm was presented by de Boor and Golub [4] and by Boley and Golub [2]. Inverse eigenvalue problems for matrices in general, and for Jacobi matrices in particular, is a very actively studied subject. In particular, we would like to mention the work described in [8], [11], [12].

The spectral peoperties of periodic Jacobi matrices are considered in Section 3 . In Section 4 we use these results to characterize the family of periodic Jacobi matrices with prescribed spectra. The results of several numerical experiments are presented in Section 5. These results demonstrate that the algorithms presented in Theorems 2.2 and 3.4 are of practical utility. In Section 6 we conclude the paper with several comments.

2. Spectral Properties of Jacobi Matrices. In this section we will consider the spectral properties of the Jacobi matrix

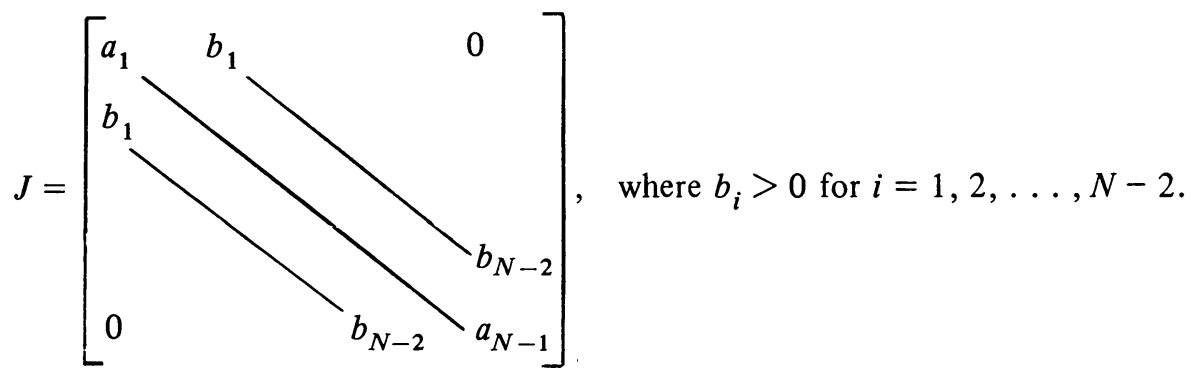

Observe that $J$ is a real, symmetric matrix. Consequently $J$ has real eigenvalues $\mu_{1}$, $\mu_{2}, \ldots, \mu_{N-1}$ and a corresponding set $Y_{1}, Y_{2}, \ldots, Y_{N-1}$ of real, orthonormal eigenvectors [16], [17]. If $Y$ denotes the matrix whose $j$ th column is $Y_{j}$, then $Y$ is an 
orthogonal matrix and

$$
J Y=Y D \text { where } D=\left[\begin{array}{c}
\mu_{N-1} \\
0
\end{array}\right]
$$

Many important relationships between the eigenvalues and eigenvectors of $J$ can be derived from the representation

$$
(\mu I-J)^{-1}=Y(\mu I-D)^{-1} Y^{T}
$$

of the resolvent of $J$. For example, by comparing the entries in row 1, column $N-1$ of (2) we obtain the identity

$$
b_{1} b_{2} \ldots b_{N-2}=\sum_{k=1}^{N-1} \frac{\omega_{J}(\mu)}{\mu-\mu_{k}} Y_{1, k} Y_{N-1, k} .
$$

Here

$$
\omega_{J}(\mu)=\operatorname{det}(\mu I-J)
$$

is the characteristic polynomial of $J$, and $Y_{i, j}$ denotes the entry of $Y$ in row $i$, column $j$. Another important identity

$$
\omega_{\tilde{J}}(\mu)=\sum_{k=1}^{N-1} \frac{\omega_{J}(\mu)}{\mu-\mu_{k}} Y_{1, k}^{2},
$$

sometimes attributed to Cauchy, is obtained from (2) when we compare the entries in row 1 , column 1 . Here $\omega \widetilde{J}$ is the characteristic polynomial of the Jacobi matrix $\widetilde{J}$ obtained from $J$ by deleting the first row and column.

Following the work of Boley and Golub, we will demonstrate that the Lanczos algorithm can be used to recover the entries of $J$ from the entries on the diagonal of $D$ and in the first row of $Y$. Before we describe this algorithm, let us introduce the following:

Definition 2.1. (a) The Jacobi matrix $J$ is characterized by the data $\{\mu, y\}$ if and only if

(1) $\mu_{1}, \mu_{2}, \ldots, \mu_{N-1}$ are the eigenvalues of $J$, and

(2) $y_{1}, y_{2}, \ldots, y_{N-1}$ are the first components of a set $Y_{1}, Y_{2}, \ldots, Y_{N-1}$ of real, orthonormal eigenvectors of $J$ corresponding to $\mu_{1}, \mu_{2}, \ldots, \mu_{N-1}$.

(b) The data $\{\mu, y\}$ are compatible if and only if

(1) $\mu_{1}, \mu_{2}, \ldots, \mu_{N-1}$ are real, distinct numbers, and

(2) $y_{1}, y_{2}, \ldots, y_{N-1}$ are real, nonzero numbers whose squares sum to one.

We feel justified in using the words "characterize" and "compatible" in this manner because the following theorem is true. 
TheOREM 2.2. Data characterizing a Jacobi matrix are compatible. Furthermore, each set of compatible data $\{\mu, y\}$ characterizes a unique Jacobi matrix $J$. The entries $(a, b)$ of this Jacobi matrix are computed by the Lanczos algorithm:

1. $b_{0}=1$;

2. $Y_{0, j}=0$ for $j=1,2, \ldots, N-1$;

3. $Y_{1, j}=y_{j}$ for $j=1,2, \ldots, N-1$;

4. For $i=1,2, \ldots, N-2$;

5. $a_{i}=\Sigma_{k=1}^{N-1} \mu_{k} Y_{k, k}^{2}$;

6. $b_{i}={ }_{+} \sqrt{\sum_{k=1}^{N-1}\left[\left(\mu_{k}-a_{i}\right) Y_{i, k}-b_{i-1} Y_{i-1, k}\right]^{2}}$;

7. $Y_{i+1, j}=\left[\left(\mu_{j}-a_{i}\right) Y_{i, j}-b_{i-1} Y_{i-1, j}\right] / b_{i}$ for $j=1,2, \ldots, N-1$;

8. Next $i$

9. $a_{N-1}=\Sigma_{k=1}^{N-1} \mu_{k} Y_{N-1, k}^{2}$.

Proof. The proof of this theorem will be presented as a sequence of three lemmas.

Lemma 2.3. Data characterizing a Jacobi matrix are compatible.

Proof. Let the Jacobi matrix $J$ be characterized by the data $\{\mu, y\}$. The $\mu_{i}$ 's are real because they are the eigenvalues of a real, symmetric matrix. Clearly the $y_{i}$ 's are real, and their squares sum to one because they are also the entries in the first row of the orthogonal matrix $Y$ in (1). Consider the limiting form of the identity (3) as $\mu$ tends to one of the eigenvalues of $J$, say $\mu_{j}$. If $\mu_{j}$ were a repeated eigenvalue, then we would be forced to conclude that $b_{1} b_{2} \cdots b_{N-2}=0$, which is impossible because each $b_{i}$ is positive. Therefore the eigenvalues of $J$ are distinct and

$$
b_{1} b_{2} \ldots b_{N-2}=\omega^{\prime}\left(\mu_{j}\right) Y_{1, j} Y_{N-1, j} \text { for } j=1,2, \ldots, N-1 .
$$

From (5) we also infer that the $y_{j}$ 's are nonzero because $y_{j}=Y_{1, j}$ for $j=1$, $2, \ldots, N-1$.

Lemma 2.4. Given compatible data $\{\mu, y\}$ the Lanczos algorithm of Theorem 2.2 computes the entries $(a, b)$ of a Jacobi matrix $J$ characterized by the data $\{\mu, y\}$.

Proof. First, we infer that the Lanczos algorithm computes the entries $(a, b)$ of some Jacobi matrix $J$ only if the value of $b_{i}$ computed in step 6 is never zero. From the compatibility of the data we infer that $b_{1}>0$. If $b_{1}, b_{2}, \ldots, b_{l-1}>0$ but $b_{l}=0$ for some $l<N-1$, then step 7 implies

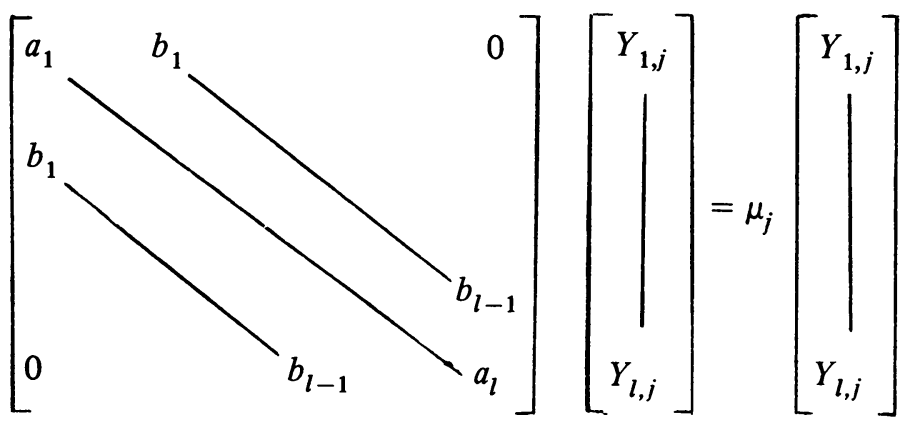


for $j=1,2, \ldots, N-1$. But this is impossible, for no matrix of order $l<N-1$ has $N-1$ distinct eigenvalues.

Second, we will demonstrate that the numbers $Y_{i, j}$, computed by this algorithm, form the entries of an orthogonal matrix $Y$. That is, the rows of $Y$ satisfy the orthonormality relations

$$
\sum_{k=1}^{N-1} Y_{i, k} Y_{j, k}=\delta_{i, j} \text { for } j=1,2, \ldots, i
$$

and $i=1,2, \ldots, N-1$. From the compatibility of the data we infer that (6) is true for $i=1$. If $(6)$ is true for $i=1,2, \ldots, l$, then the following argument demonstrates that it is also true for $i=l+1$. Clearly steps 6 and 7 imply that (6) is true for $j=l+1$. For $j \leqslant l$ step 7 implies that

$$
\sum_{k=1}^{N-1} Y_{l+1, k} Y_{j, k}=\frac{1}{b_{l}}\left[\sum_{k=1}^{N-1} \mu_{k} Y_{l, k} Y_{j, k}-a_{l} \delta_{l, j}-b_{l-1} \delta_{l-1, j}\right]
$$

Since step 5 was executed, the right side of this equality is zero for $j=l$. The right side of this equality is also zero for $j<l$ because step 7 implies that

$$
\sum_{k=1}^{N} \mu_{k} Y_{l, k} Y_{j, k}=\sum_{k=1}^{N-1} Y_{l, k}\left[b_{j-1} Y_{j-1, k}+a_{j} Y_{j, k}+b_{j} Y_{j+1, k}\right]=b_{j} \delta_{l, j+1}
$$

Third, we will demonstrate that the data $\{\mu, y\}$ characterizes $J$. It will be sufficient to prove that the matrices $J, Y$ constructed by this algorithm satisfy (1). Step 7 implies that $J Y=Y D$ if we can show that the numbers

$$
Y_{N, j} \equiv\left(\mu_{j}-a_{i}\right) Y_{N-1, j}-b_{N-2} Y_{N-2, j}
$$

are zero for $j=1,2, \ldots, N-1$. The techniques presented in the previous paragraph can be used to demonstrate that

$$
\sum_{k=1}^{N-1} Y_{N, k} Y_{j, k}=0 \quad \text { for } j=1,2, \ldots, N-1
$$

Since the rows of $Y$ form a real, orthonormal basis for $\mathbf{R}^{N-1}$, we infer that

$$
Y_{N, j}=0 \quad \text { for } j=1,2, \ldots, N-1 \text {. }
$$

Lemma 2.5. Each set of compatible data characterizes at most one Jacobi matrix.

Proof. Let $\hat{J}$ be any Jacobi matrix characterized by the compatible data $\{\mu, y\}$. Then $y_{1}, y_{2}, \ldots, y_{N-1}$ are the first components of a set $\hat{Y}_{1}, \hat{Y}_{2}, \ldots, \hat{Y}_{N-1}$ of real, orthonormal eigenvectors of $\hat{J}$ corresponding to the eigenvalues $\mu_{1}, \mu_{2}, \ldots, \mu_{N-1}$. If $\hat{Y}$ denotes the matrix whose $j$ th column is $\hat{Y}_{j}$, then $\hat{Y}$ is an orthogonal matrix and 


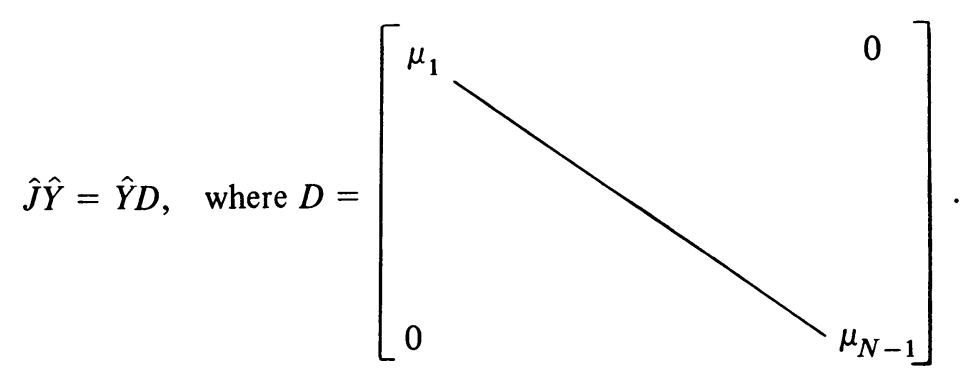

We will now prove that the entries $(\hat{a}, \hat{b})$ of $\hat{J}$ are identical to the entries $(a, b)$ of the Jacobi matrix $J$ computed by the Lanczos algorithm presented in Theorem 2.2.

The entries $\hat{Y}_{i, j}$ of $\hat{Y}$ satisfy the orthonormality relations

$$
\sum_{k=1}^{N-1} \hat{Y}_{i, k} \hat{Y}_{j, k}=\delta_{i, j} \quad \text { for } i, j=1,2, \ldots, N-1,
$$

because $\hat{Y} \hat{Y}^{T}=I$. The entries $\hat{Y}_{i, j}$ of $\hat{Y}$ also satisfy the recurrence relation

$$
\hat{b}_{i-1} \hat{Y}_{i-1, j}+\hat{a}_{i} \hat{Y}_{i, j}+\hat{b}_{i} \hat{Y}_{i+1, j}=\mu_{j} \hat{Y}_{i, j} \text { for } i, j=1,2, \ldots, N-1,
$$

with

$$
\hat{Y}_{0, j}=0 \quad \text { and } \quad \hat{Y}_{N, j}=0 \text { for } j=1,2, \ldots, N-1 \text {, }
$$

because $\hat{J} \hat{Y}=\hat{Y} D$. When the recurrence relation is multiplied by $\hat{Y}_{i, j}$ and the result is summed over $j$, we find, using the orthonormality relations, that

$$
\hat{a}_{i}=\sum_{k=1}^{N-1} \mu_{k} \hat{Y}_{i, k}^{2}
$$

The recurrence relation also implies that

$$
\hat{Y}_{i+1, j}=\frac{1}{\hat{b}_{i}}\left[\left(\mu_{j}-\hat{a}_{i}\right) \hat{Y}_{i, j}-\hat{b}_{i-1} \hat{Y}_{i-1, j}\right] \text { for } j=1,2, \ldots, N-1,
$$

and, when this identity is squared and the result is summed over $j$, the orthonormality relations imply that

$$
\hat{b}_{i}=\sqrt{\sum_{k=1}^{N-1}\left[\left(\mu_{k}-\hat{a}_{i}\right) \hat{Y}_{i, k}-\hat{b}_{i-1} \hat{Y}_{i-1, k}\right]^{2}}
$$

Starting with the fact that $\hat{Y}_{1, j}=y_{j}$ for $j=1,2, \ldots, N-1$, it is easily shown, by following the sequence of computations presented in the Lanczos algorithm of Theorem 2.2 , that the entries $(\hat{a}, \hat{b})$ of $\hat{J}$ are identical to the entries $(a, b)$ of $J$.

The statement and proof of Theorem 2.2 certainly would not surprise anyone familiar with this branch of linear algebra. Lemma 2.1 was presented because it contains the important identity (5). Lemma 2.2 was presented because it demonstrates that, given compatible data, the Lanczos algorithm cannot prematurely terminate by producing a $b_{i}$ in step 6 which is zero, a point not mentioned in the paper by Boley and Golub [2]. Lemma 2.3 was presented because it shows how one could have deduced the Lanczos algorithm from the eigenidentity

$$
J Y=Y D .
$$


Indeed, by using Lemma 2.3 as an outline, it is possible to deduce the generalization of the Lanczos algorithm needed to solve the inverse eigenvalue problem posed by Boley and Golub [2]. Interestingly enough, the generalization so derived is not the block Lanczos algorithm Boley and Golub use to solve their inverse eigenvalue problem.

We have chosen to characterize a Jacobi matrix $J$ by the data $\{\mu, y\}$ for two reasons. First, this characterization makes it possible to simply describe an algorithm which solves the inverse eigenvalue problem for periodic Jacobi matrices. Second, this characterization makes it possible to generalize Definition 2.1 and Theorem 2.2 to the same class of band matrices considered by Boley and Golub [2] .

The Lanczos algorithm of Theorem 2.2 can also be used to solve the inverse eigenvalue problems considered in [4], [11], [12]. Recall that $\omega_{\tilde{J}}$ is the characteristic polynomial of the Jacobi matrix $\widetilde{J}$ obtained from $J$ by deleting the first row and column. Given the eigenvalues of $\widetilde{J}$ and $J$, we can recover the data $\{\mu, y\}$ characterizing $J$ from the identity

$$
\omega_{\widetilde{J}}\left(\mu_{j}\right)=\omega_{J}^{\prime}\left(\mu_{j}\right) y_{j}^{2} \quad \text { for } j=1,2, \ldots, N-1,
$$

derived from (4).

It is worth mentioning that each $y_{j}$ in the data $\{\mu, y\}$ characterizing $J$ is unique to within a sign. For if $Y_{j}$ is an eigenvector of $J$ corresponding to the eigenvalue $\mu_{j}$, then so is $-Y_{j}$. Consequently $y_{j}$ may be taken to be either $+Y_{1, j}$ or $-Y_{1, j}$. More precisely, we may say that there is a one-to-one correspondence between the set of Jacobi matrices $J$ and the set of compatible data $\{\mu, y\}$ having each $y_{j}$ positive.

3. Spectral Properties of Periodic Jacobi Matrices. In this section we will consider the spectral properties of the periodic Jacobi matrix

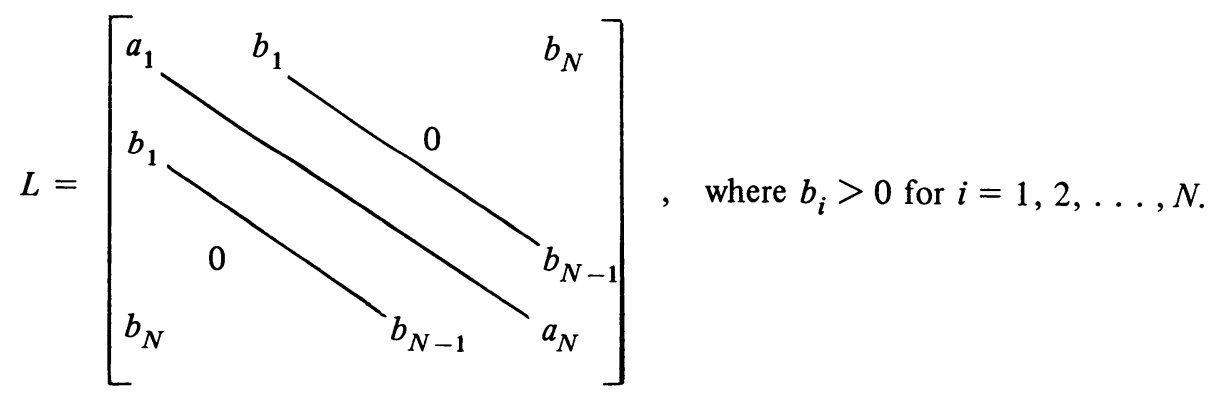

Throughout this section we will use $J$ to represent the Jacobi matrix obtained by deleting from $L$ the last row and column.

Observe that $L$ is a real, symmetric matrix. Consequently $L$ has real eigenvalues and a corresponding set of real, orthonormal eigenvectors [16], [17]. Let $z$ be an eigenvector of $L$ corresponding to the eigenvalue $\lambda$. From the eigenidentity

$$
L z=\lambda z,
$$

we infer that the components $z_{i}$ of $z$ form a nontrivial solution of the recurrence relation $\left(b_{0} \equiv b_{N}\right)$

$$
b_{i-1} z_{i-1}+a_{i} z_{i}+b_{i} z_{i+1}=\lambda z_{i} \text { for } i=1,2, \ldots, N,
$$


which satisfies the boundary conditions

$$
z_{N}=z_{0} \text { and } z_{N+1}=z_{1} .
$$

By analogy with Floquet theory, which analyzes the analogous problem for ordinary differential equations [14], let us consider the nontrivial solutions of this recurrence relation which satisfy the boundary conditions

$$
z_{N}=\rho z_{0} \text { and } z_{N+1}=\rho z_{1} .
$$

Here the parameter $\rho$ is called the Floquet multiplier of the solution $z$. When $\rho=0$ there are no nontrivial solutions, while for $\rho \neq 0$ a nontrivial solution exists if and only if $\lambda$ is an eigenvalue of the matrix

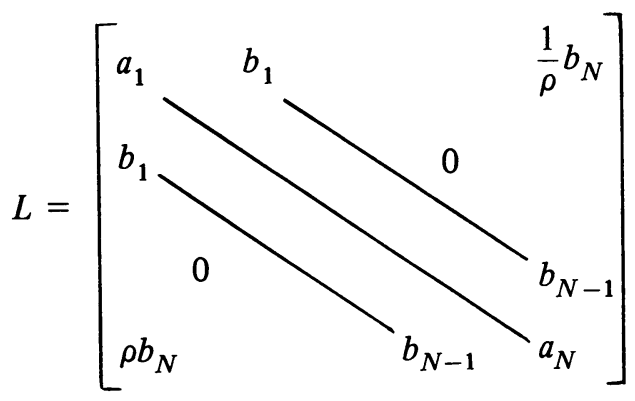

With these facts in mind let us introduce the following

Definition 3.1. Let the $J$ be characterized by the data $\{\mu, y\}$ and have $\omega_{J}$ as its characteristic polynomial. Then the Floquet multipliers $\rho_{1}, \rho_{2}, \ldots, \rho_{N-1}$ of $L$ corresponding to $\mu_{1}, \mu_{2}, \ldots, \mu_{N-1}$ are the numbers defined by the relation

$$
b_{1} b_{2} \cdots b_{N}=-\rho_{j} \omega_{J}^{\prime}\left(\mu_{j}\right) b_{N}^{2} y_{j}^{2} \quad \text { for } j=1,2, \ldots, N-1 .
$$

Some of the interesting spectral properties of $L_{\rho}$ are described in the following

THEOREM 3.2. The characteristic polynomial of $L_{\rho}$ admits the representation

$$
\operatorname{det}\left(\lambda I-L_{\rho}\right)=b_{1} b_{2} \cdots b_{N}\left\{\Delta(\lambda)-\left(\rho+\frac{1}{\rho}\right)\right\},
$$

where $\Delta$, called the discriminant of $L$, is independent of $\rho$. The Floquet multipliers $\rho_{1}, \rho_{2}, \ldots, \rho_{N-1}$ of $L$ corresponding to the eigenvalues $\mu_{1}, \mu_{2}, \ldots, \mu_{N-1}$ of $J$ satisfy the relation

$$
\Delta\left(\mu_{j}\right)=\rho_{j}+1 / \rho_{j} \text { for } j=1,2, \ldots, N-1 .
$$

Furthermore, the eigenvalues $\lambda_{1}, \lambda_{2}, \ldots, \lambda_{N}$ of $L$ are real and can be ordered so that

$$
\lambda_{1}>\lambda_{2} \geqslant \lambda_{3}>\lambda_{4} \geqslant \lambda_{5}>\ldots \text {. }
$$

Proof. Using elementary properties of determinants, it is not hard to demonstrate that

$$
\frac{d}{d \rho} \operatorname{det}\left(\lambda I-L_{\rho}\right)=-b_{1} b_{2} \cdots b_{N}\left(1-\frac{1}{\rho^{2}}\right)
$$


When both sides are integrated with respect to $\rho$, we find that

$$
\operatorname{det}\left(\lambda I-L_{\rho}\right)=b_{1} b_{2} \cdots b_{N}\left\{\Delta(\lambda)-\left(\rho+\frac{1}{\rho}\right)\right\} .
$$

Of course, the constant of integration $b_{1} b_{2} \ldots b_{N} \Delta(\lambda)$ is necessarily independent of $\rho$.

Let $J$ be characterized by the data $\{\mu, y\}$. Then $y_{1}, y_{2}, \ldots, y_{N-1}$ are the first components of a set $Y_{1}, Y_{2}, \ldots, Y_{N-1}$ of real, orthonormal eigenvectors of $J$ corresponding to its eigenvalues $\mu_{1}, \mu_{2}, \ldots, \mu_{N-1}$. Let $Y_{i, j}$ denote the $i$ th component of $Y_{j}$. From the definition (7) of the Floquet multipliers and the identity (5), we infer that

$$
\rho_{j}=-\frac{b_{N-1} Y_{N-1, j}}{b_{N} Y_{1, j}} \quad \text { for } j=1,2, \ldots, N-1 \text {, }
$$

and so

$$
L_{\rho_{j}}\left[\begin{array}{c}
Y_{j} \\
0
\end{array}\right]=\mu_{j}\left[\begin{array}{c}
Y_{j} \\
\\
0
\end{array}\right] \text { for } j=1,2, \ldots, N-1 \text {. }
$$

Consequently $\mu_{j}$ is an eigenvalue of $L_{\rho_{j}}$ for $j=1,2, \ldots, N-1$, and we infer from (8) that (9) is true.

From the definition (7) of the Floquet multipliers, we deduce that

$$
\omega_{J}^{\prime}\left(\mu_{j}\right) \rho_{j}<0 \text { for } j=1,2, \ldots, N-1 \text {. }
$$

When the eigenvalues $\mu_{1}, \mu_{2}, \ldots, \mu_{N-1}$ of $J$ are ordered so that $\mu_{1}>\mu_{2}>\cdots>$ $\mu_{N-1}$, we infer from (9) that

$$
(-1)^{j} \Delta\left(\mu_{j}\right) \geqslant 2 \text { for } j=1,2, \ldots, N-1,
$$

because the magnitude of $\rho+1 / \rho$ is never less than two. Consequently the eigenvalues $\lambda_{1}, \lambda_{2}, \ldots, \lambda_{N}$ of $L$, which are the roots of $\Delta=2$, are real and can be ordered so that $\lambda_{1}>\lambda_{2} \geqslant \lambda_{3}>\lambda_{4} \geqslant \lambda_{5}>\cdots$, because the coefficient $\left(b_{1} b_{2} \cdots b_{N}\right)^{-1}$ of $\lambda^{N}$ in $\Delta$ is positive.

A typical discriminant of a Jacobi matrix $L$ of order $N=6$ is illustrated in Figure 1. In this figure we have depicted the relationship between the eigenvalues $\lambda_{1}$, $\lambda_{2}, \ldots, \lambda_{N}$ of $L$ and the Floquet multipliers $\rho_{1}, \rho_{2}, \ldots, \rho_{N-1}$ of $L$ corresponding to the eigenvalues $\mu_{1}, \mu_{2}, \ldots, \mu_{N-1}$ of $J$.

Definition 3.3. (a) The periodic Jacobi matrix $L$ is characterized by the data $\{A, B, \mu, \rho\}$ if and only if

(1) $A=a_{1}+a_{2}+\cdots+a_{N}$,

(2) $B=b_{1} b_{2} \cdots b_{N}$,

(3) $\mu_{1}, \mu_{2}, \ldots, \mu_{N-1}$ are the eigenvalues of $J$, and

(4) $\rho_{1}, \rho_{2}, \ldots, \rho_{N-1}$ are the Floquet multipliers of $L$ corresponding to $\mu_{1}$, $\mu_{2}, \ldots, \mu_{N-1}$. 
(b) The data $\{A, B, \mu, \rho\}$ are compatible if and only if

(1) $A$ is a real number,

(2) $B$ is a real, positive number,

(3) $\mu_{1}, \mu_{2}, \ldots, \mu_{N-1}$ are real, distinct numbers, and

(4) $\rho_{1}, \rho_{2}, \ldots, \rho_{N-1}$ are real numbers which satisfy $\omega^{\prime}\left(\mu_{j}\right) \rho_{j}<0$ for $j=1$, $2, \ldots, N-1$ with $\omega(\mu)=\left(\mu-\mu_{1}\right)\left(\mu-\mu_{2}\right) \cdots\left(\mu-\mu_{N-1}\right)$.

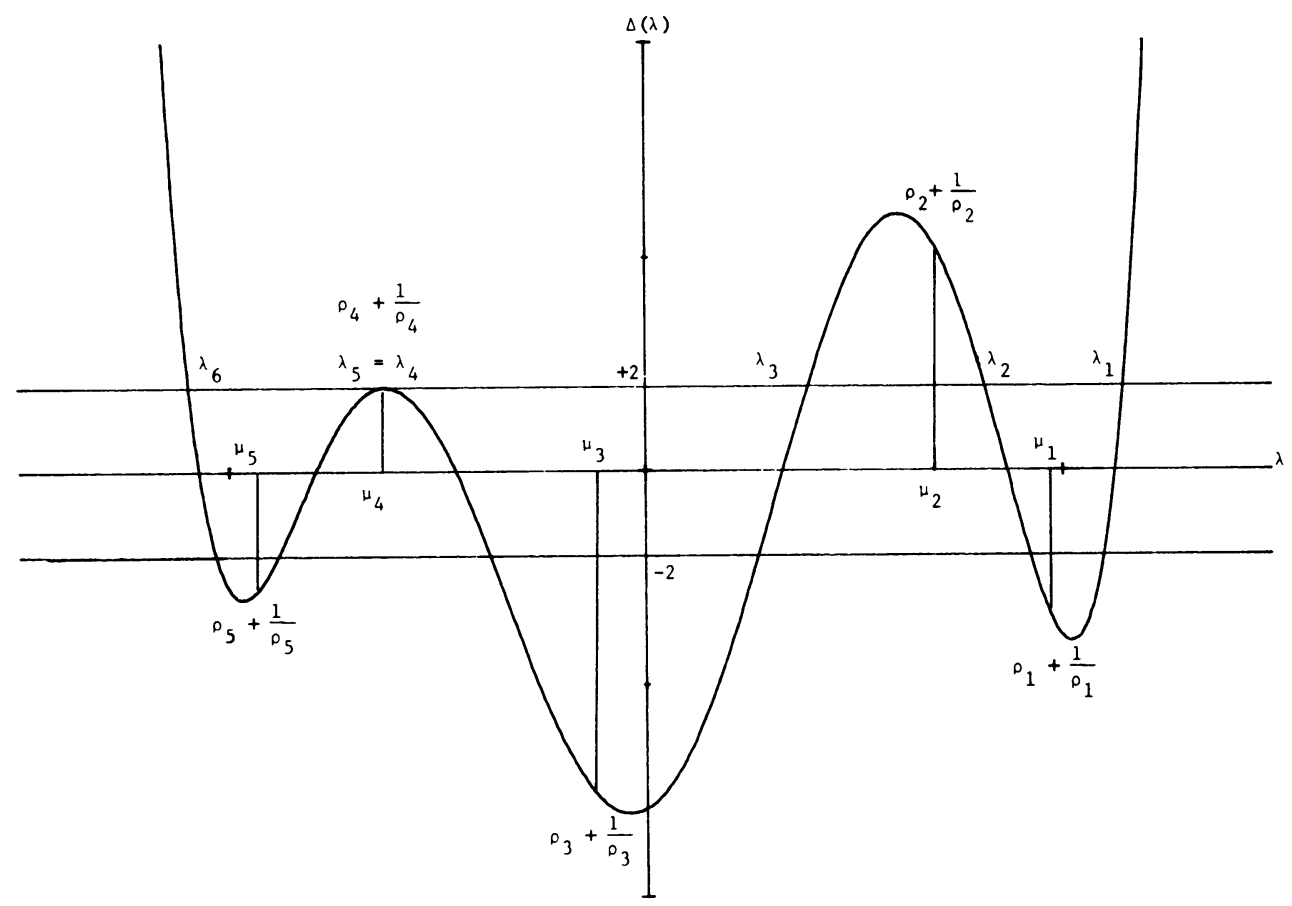

FIGURE 1

Plot of a typical discriminant, $N=6$

We feel justified in using the words "characterize" and "compatible" in this manner because the following theorem is true.

THEOREM 3.4. Data characterizing a periodic Jacobi matrix are compatible.

Furthermore, each set of compatible data $\{A, B, \mu, \rho\}$ characterizes a unique periodic Jacobi matrix $L$. The entries $(a, b)$ of this periodic Jacobi matrix $L$ are computed by the algorithm:

1. $b_{N}={ }_{+} \sqrt{-\sum_{k=1}^{N-1}\left(B / \rho_{k} \omega^{\prime}\left(\mu_{k}\right)\right)}$;

2. $y_{j}=\left(1 / b_{N}\right)_{+} \sqrt{-\left(B / \rho_{j} \omega^{\prime}\left(\mu_{j}\right)\right)}$ for $j=1,2, \ldots, N-1$;

3. Recover the Jacobi matrix $J$ characterized by the data $\{\mu, y\}$;

4. $b_{N-1}=B / b_{1} b_{2} \cdots b_{N-2} b_{N}$;

5. $a_{N}=A-\left(a_{1}+a_{2}+\cdots+a_{N-1}\right)$;

where $\omega(\mu)-\left(\mu-\mu_{1}\right)\left(\mu-\mu_{2}\right) \cdots\left(\mu-\mu_{N-1}\right)$.

Proof. The proof of this theorem will be presented as a sequence of three lemmas. 


\section{Lemma 3.5. Data characterizing a periodic Jacobi matrix are compatible.}

Proof. Let the data $\{A, B, \mu, \rho\}$ characterize the periodic Jacobi matrix $L$. Clearly $A$ is a real number and $B$ is a real, positive number. The $\mu_{j}$ 's are real, distinct numbers since they are the eigenvalues of the Jacobi matrix $J$. The definition (7) of the $\rho_{j}$ 's makes it obvious that they are real, nonzero numbers which satisfy $\omega^{\prime}\left(\mu_{j}\right) \rho_{j}<0$ for $j=1,2, \ldots, N-1$ because $\omega$ is also the characteristic polynomial of $J$.

Lemma 3.6. Given compatible data $\{A, B, \mu, \rho\}$, the algorithm of Theorem 3.4 computes the entries $(a, b)$ of a periodic Jacobi matrix $L$ characterized by the data $\{A, B, \mu, \rho\}$.

Proof. The data $\{\mu, y\}$ used in step 3 in the algorithm of Theorem 3.4 are compatible, therefore it is clear that this algorithm computes the entries $(a, b)$ of some periodic Jacobi matrix $L$. Let $L$ be characterized by the data $\{\hat{A}, \hat{B}, \hat{\mu}, \hat{\rho}\}$. From steps 4 and 5 of this algorithm it is clear that $\hat{A}=A$ and $\hat{B}=B$. In view of Theorem 2.2 we know that $J$ is characterized by the data $\{\mu, y\}$. Therefore $\hat{\mu}_{j}=\mu_{j}$ for $j=1$, $2, \ldots, N-1$, and from the definition of the Floquet multipliers we know that

$$
B=-\hat{\rho}_{j} \omega^{\prime}\left(\mu_{j}\right) b_{N}^{2} y_{j}^{2} \quad \text { for } j=1,2, \ldots, N-1 .
$$

Step 2 of this algorithm therefore implies that $\hat{\rho}_{j}=\rho_{j}$ for $j=1,2, \ldots, N-1$.

LEMMA 3.7. Each set of compatible data characterizes at most one periodic Jacobi matrix.

Proof. Let $\hat{L}$ be any periodic Jacobi matrix characterized by the data $\{A, B, \mu, \rho\}$. Let the Jacobi matrix $\hat{J}$, obtained from $\hat{L}$ by deleting the last row and column, be characterized by the data $\{\mu, \hat{y}\}$. As pointed out at the end of Section 2, we may assume that each $\hat{y}_{j}$ is positive. We will now prove that the entries $(\hat{a}, \hat{b})$ of $\hat{L}$ are identical to the entries $(a, b)$ of the periodic Jacobi matrix $L$ constructed by the algorithm of Theorem 3.4.

By definition the Floquet multipliers $\rho_{1}, \rho_{2}, \ldots, \rho_{N-1}$ of $\hat{L}$ corresponding to $\mu_{1}, \mu_{2}, \ldots, \mu_{N-1}$, satisfy the relationship

$$
B=-\rho_{j} \omega^{\prime}\left(\mu_{j}\right) \hat{b}_{N}^{2} \hat{y}_{j}^{2} \quad \text { for } j=1,2, \ldots, N-1 .
$$

The sum of the squares of the $\hat{y}_{j}$ 's equals one because the data $\{\mu, \hat{y}\}$ is compatible, therefore

$$
\hat{b}_{N}^{2}=-\sum_{k=1}^{N-1} \frac{B}{\rho_{k} \omega^{\prime}\left(\mu_{k}\right)}
$$

and

$$
\hat{y}_{j}^{2}=-\frac{B}{b_{N}^{2} \rho_{j} \omega^{\prime}\left(\mu_{j}\right)} \quad \text { for } j=1,2, \ldots, N-1 .
$$

In view of steps 1 and 2 of this algorithm, we infer that $\hat{b}_{N}=b_{N}$ and $\hat{y}_{j}=y_{j}$ for $j=1,2, \ldots, N-1$. Since $J$ and $\hat{J}$ are characterized by the same data, Theorem 2.2 implies that $J=\hat{J}$. Finally, steps 4 and 5 of this algorithm imply that $\hat{b}_{N-1}=b_{N-1}$ and $\hat{a}_{N}=a_{N}$. 
Observe that Theorem 3.4 recovers the periodic Jacobi matrix $L$ by first recovering the Jacobi matrix $J$. This idea was first proposed by van Moerbeke [15], although his algorithm is known to be numerically unstable. The algorithm presented in Theorem 3.4 inherits its numerical stability from the stability of the Lanczos algorithm used in step 3.

The algorithm presented in Theorem 3.4 is different from the algorithm proposed by Boley and Golub [3]. In particular, Boley and Golub only consider recovering those periodic Jacobi matrices characterized by data $\{A, B, \mu, \rho\}$ for which $\left|\rho_{j}\right| \neq 1$ for $j=1,2, \ldots, N-1$. For example, their algorithm could not recover the periodic Jacobi matrix

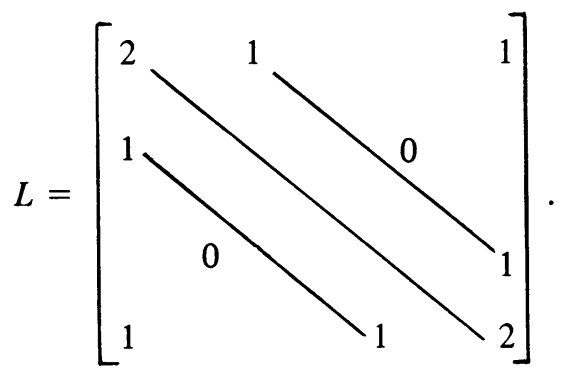

4. Periodic Jacobi Matrices With Prescribed Spectra. With these basic facts established let us now consider how we can characterize the family of periodic Jacobi matrices whose eigenvalues are $\lambda_{1}, \lambda_{2}, \ldots, \lambda_{N}$.

Let $L$ be a periodic Jacobi matrix characterized by the data $\{A, B, \mu, \rho\}$. Then $\lambda_{1}, \lambda_{2}, \ldots, \lambda_{N}$ are the eigenvalues of $L$ if and only if the discriminant $\Delta$ of $L$ admits the representation

$$
\Delta(\lambda)=2+\frac{1}{B}\left(\lambda-\lambda_{1}\right)\left(\lambda-\lambda_{2}\right) \cdots\left(\lambda-\lambda_{N}\right) .
$$

Therefore the problem of characterizing the family of periodic Jacobi matrices with prescribed spectra is intimately related to the problem of characterizing the family of periodic Jacobi matrices with prescribed discriminant.

Definition 4.1. For each polynomial $p$ let $F(p)$ denote the family of periodic Jacobi matrices whose discriminant is $p$.

The problem of characterizing which periodic Jacobi matrices belong to $F(p)$ is answered in the following:

Theorem 4.2. Let $p$ by a polynomial of degree $N$. The data $\{A, B, \mu, \rho\}$ characterizes a member of $F(p)$ if and only if

(1) the data $\{A, B, \mu, \rho\}$ are compatible,

(2) $p(\lambda)=(1 / B)\left[\lambda^{N}-A \lambda^{N-1}+\right.$ lower powers of $\left.\lambda\right]$, and

(3) $p\left(\mu_{j}\right)=\rho_{j}+1 / \rho_{j}$ for $j=1,2, \ldots, N-1$.

Furthermore, $F(p)$ is nonempty if and only if

(4) the coefficient of $\lambda^{N}$ in $p(\lambda)$ is positive, and

(5) $p$ has local extrema at $N-1$ distinct points $v_{1}>v_{2}>\cdots>v_{N-1}$ with $(-1)^{j} p\left(v_{j}\right) \geqslant 2$ for $j=1,2, \ldots, N-1$. 
Proof. If the data $\{A, B, \mu, \rho\}$ characterizes a member of $F(p)$, then Theorems 3.2 and 3.4 demonstrate that conditions (1), (2), (3) are satisfied. Now suppose the data $\{A, B, \mu, \rho\}$ satisfies (1), (2), (3). Let $\Delta$ be the discriminant of the periodic Jacobi matrix characterized by $\{A, B, \mu, \rho\}$. Now

$$
q=\Delta-p
$$

is a polynomial of degree $N-2$ because the coefficients of $\lambda^{N}, \lambda^{N-1}$ in $\Delta(\lambda), p(\lambda)$ are identical. Theorem 3.2 implies that

$$
q\left(\mu_{j}\right)=0 \text { for } j=1,2, \ldots, N-1,
$$

and so $q \equiv 0$ because the only polynomial of degree $N-2$ which is zero at $N-1$ distinct points is the trivial polynomial. Therefore, the data $\{A, B, \mu, \rho\}$ characterizes a member of $F(p)$.

If $F(p)$ is nonempty, then conditions (2), (3) and the mean-value theorem can be used to demonstrate that conditions (4), (5) are satisfied. Let us now suppose that $p$ satisfies conditions (4), (5). Determine $A, B$ so that

$$
p(\lambda)=\frac{1}{B}\left[\lambda^{N}-A \lambda^{N-1}+\text { lower powers of } \lambda\right],
$$

and define $\rho_{1}, \rho_{2}, \ldots, \rho_{N-1}$ to be solutions of

$$
p\left(v_{j}\right)=\rho_{j}+\frac{1}{\rho_{j}} \quad \text { for } j=1,2, \ldots, N-1 .
$$

Then the data $\{A, B, \mu, \rho\}$ are compatible and from (1), (2), (3) we infer that the data $\{A, B, \mu, \rho\}$ characterizes a member of $F(p)$.

Using Theorem 4.2 , we can now characterize the family of Jacobi matrices having $\lambda_{1}, \lambda_{2}, \ldots, \lambda_{N}$ as its eigenvalues.

THEOREM 4.3. The periodic Jacobi matrix $L$ has $\lambda_{1}, \lambda_{2}, \ldots, \lambda_{N}$ as its eigenvalues if and only if

$$
L \in \bigcup_{B>0} F\left(\Delta_{B}\right)
$$

where

$$
\Delta_{B}(\lambda)=2+\frac{1}{B}\left(\lambda-\lambda_{1}\right)\left(\lambda-\lambda_{2}\right) \cdots\left(\lambda-\lambda_{N}\right) .
$$

Furthermore, there is a family of periodic Jacobi matrices with $\lambda_{1}, \lambda_{2}, \ldots, \lambda_{N}$ as its eigenvalues if and only if the numbers can be rearranged so that $\lambda_{1}>\lambda_{2} \geqslant \lambda_{3}>\lambda_{4} \geqslant$ $\lambda_{5}>\ldots$.

Parts of Theorems 4.2 and 4.3 appear in the work of van Moerbeke [15]. For example, in Theorem 2.1 of [15] van Moerbeke parametrizes the family of periodic Jacobi matrices having $\lambda_{1}, \lambda_{2}, \ldots, \lambda_{N}$ as their eigenvalues provided there is at least one periodic Jacobi matrix with these eigenvalues.

5. Numerical Experiments. We will now present the results of several numerical experiments. These experiments were carried out on a UNIVAC 1110 in single-precision floating-point binary arithmetic (27 bit mantissa) using FORTRAN versions of the algorithms presented in Theorems 2.2 and 3.4. 
In the first experiment we tested the algorithm presented in Theorem 2.2. The results of this experiment are presented in Table 1. Observe that this algorithm has difficulty in recovering the Jacobi matrix described in Example 3.

Experiment 1. Test the algorithm for Jacobi matrices.

1. Select a Jacobi matrix $J$ of order $N-1$.

2. Compute the data $\{\mu, y\}$ characterizing $J[17],[18]$ :

(a) use bisection to compute the $\mu_{j}$ 's and

(b) use inverse iteration to compute the $y_{j}$ 's.

3. Use the algorithm presented in Theorem 2.2 to reconstruct the Jacobi matrix $\hat{J}$ characterized by the data $\{\mu, y\}$.

4. Output the error $\|J-\hat{J}\|$, where $\|A\|=\max _{i, j}\left|a_{i, j}\right|$.

In the second experiment we tested the algorithm presented in Theorem 3.4. The results of this experiment are presented in Table 2. Observe that the Jacobi matrices used in the examples of Experiment 1 are obtained by deleting the last row and column from the periodic Jacobi matrices used in the corresponding examples of Experiment 2.

Experiment 2. Test the algorithm for periodic Jacobi matrices.

1. Select a periodic Jacobi matrix $L$ of order $N$.

2. Compute the data $\{A, B, \mu, \rho\}$ characterizing $L$ :

(a) $A=a_{1}+a_{2}+\cdots+a_{N}$,

(b) $B=b_{1} b_{2} \cdots b_{N}$,

(c) compute the data $\{\mu, y\}$ characterizing $J$ as described in step 2 of Experiment 1 ,

(d) compute the $\rho_{j}$ 's using (7).

3. Use the algorithm presented in Theorem 3.4 to reconstruct the periodic Jacobi matrix $L$ characterized by the data $\{A, B, \mu, \rho\}$.

4. Output the error $\|L-\hat{L}\|$, where $\|A\|=\max _{i, j}\left|a_{i, j}\right|$.

TABLE 1. Results of Experiment 1

Example 1:

$\begin{array}{ll}A(I)=-2 & I=1, \ldots, N-1 \\ B(I)=1 & I=1, \ldots, N-2 \\ N & \text { Error } \\ & \\ 5 & 4 \times 10^{-8} \\ 10 & 2 \times 10^{-7} \\ 15 & 5 \times 10^{-7} \\ 20 & 2 \times 10^{-7} \\ 25 & 2 \times 10^{-7} \\ 30 & 6 \times 10^{-7}\end{array}$


Example 2:

$A(I)=(N+1-I) / N-2$
$B(I)=1-(N-I) / N$
$N$
5
10
15
20
25
30

$$
\begin{aligned}
& I=1, \ldots, N-1 \\
& I=1, \ldots, N-2 \\
& \quad \text { Error } \\
& 4 \times 10^{-8} \\
& 1 \times 10^{-7} \\
& 4 \times 10^{-7} \\
& 3 \times 10^{-7} \\
& 3 \times 10^{-7} \\
& 9 \times 10^{-7}
\end{aligned}
$$

Example 3:

$\begin{array}{ll}A(I)=I / N-2 & I=1, \ldots, N-1 \\ B(I)=1-I / N & I=1, \ldots, N-2 \\ N & \text { Error } \\ 5 & 1 \times 10^{-7} \\ 10 & 3 \times 10^{-7} \\ 15 & 2 \times 10^{-4} \\ 20 & 2 \times 10^{0} \\ 25 & 2 \times 10^{0} \\ 30 & 1 \times 10^{0}\end{array}$

TABLE 2. Results of Experiment 2

Example 1:

$\begin{array}{ll}A(I)=-2 & I=1, \ldots, N-1 \\ B(I)=1 & I=1, \ldots, N-2 \\ A(N)=0 & \\ B(N-1)=B(N)=1 & \\ N & \text { Error } \\ 5 & 9 \times 10^{-8} \\ 10 & 5 \times 10^{-7} \\ 15 & 1 \times 10^{-6} \\ 20 & 2 \times 10^{-6} \\ 25 & 3 \times 10^{-6} \\ 30 & 5 \times 10^{-6}\end{array}$


Example 2:

$\begin{array}{ll}A(I)=(N+1-I) / N-2 & I=1, \ldots, N-1 \\ B(I)=1-(N-I) / N & I=1, \ldots, N-2 \\ A(N)=0 & \\ B(N-1)=B(N)=1 & \\ N & \text { Error } \\ 5 & 1 \times 10^{-7} \\ 10 & 2 \times 10^{-7} \\ 15 & 4 \times 10^{-7} \\ 20 & 3 \times 10^{-7} \\ 25 & 6 \times 10^{-7} \\ 30 & 4 \times 10^{-7}\end{array}$

Example 3:

$$
\begin{array}{ll}
A(I)=I / N-2 & I=1, \ldots, N-1 \\
B(I)=1-I / N & I=1, \ldots, N-2 \\
A(N)=0 & \\
B(N-1)=B(N)=1 &
\end{array}
$$

$N$

\section{5}

10

15

20

25

30

\section{Error}

$4 \times 10^{-8}$

$1 \times 10^{-7}$

$1 \times 10^{-3}$

$2 \times 10^{0}$

$4 \times 10^{0}$

$5 \times 10^{0}$

In both of these experiments we have not worked with matrices of order $N$ greater than thirty. In Example 2 of Experiment 2 some of the components of $y$ in the data $\{\mu, y\}$ characterizing $J$ become smaller as $N$ increases. For example, the smallest component of $y$ changes from $O\left(10^{-9}\right)$ for $N=15$ to $O\left(10^{-20}\right)$ for $N=30$. Since the Floquet multipliers $\rho_{j}$ depend on the squares of the corresponding $y_{j}$, we would therefore run into underflow problems. The immediate remedy for this underflow problem is to compute the logarithms of each $\rho_{j}$ instead of $\rho_{j}$. However, underflow also occurs in the computation of the $y_{j}$ 's when $N$ is greater than fifty-five.

6. Comments. It is interesting to note that the Lanczos algorithm of Theorem 2.2 is used in some versions of the implicit shift $Q R$ algorithm [16]. These versions of the $Q R$ algorithm make use of the fact that if $B=Q A Q^{H}$, where $B$ is an unreduced upper Hessenberg matrix and $Q$ is a unitary matrix, then the entries of $B$ and $Q$ are uniquely determined from the entries of $A$ and the entries in the first row of $Q$. In our application we have $A=D, Q=Y$, and $B=J$ ! 
We can also recover the Jacobi matrix $J$ from the eigenvalues and the last components of the corresponding real, orthonormal eigenvectors of $J$. To understand why let us consider the permutation matrix

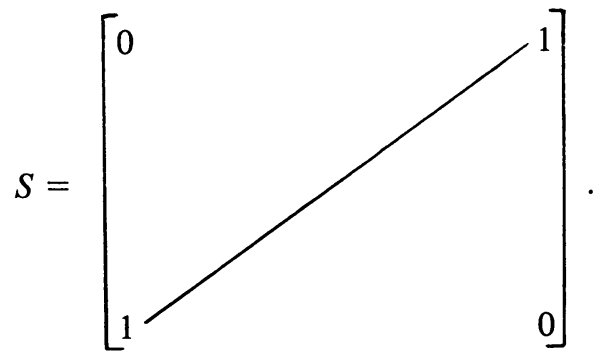

We find that $S^{2}=I$, therefore from (1) we deduce that

$$
(S J S)(S Y)=(S Y) D \text {. }
$$

Consequently the algorithm presented in Theorem 2.2 states that the entries of

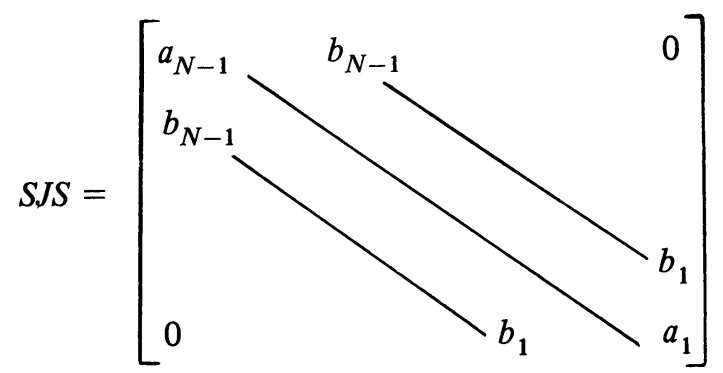

can be recovered from the entries of $D$ and the entries in the first row of $S Y$, that is the last row of $Y$. There is also an identity analogous to (4) which relates the elements in the last row of $Y$ to the eigenvalues of $J$ and the eigenvalues of the Jacobi matrix obtained from $J$ by deleting the last row and column. This identity is easily obtained by comparing the entries in row $N-1$, column $N-1$ of (2).

The paper by Golub and Welsch [10] outlines how one can modify the usual $Q R$ algorithm and directly compute the data $\{\mu, y\}$ characterizing a Jacobi matrix $J$. This paper is also important because it presents a matrix version of the celebrated GelfandLevitan solution to the inverse eigenvalue problem for a class of Sturm-Liouville problems.

The paper by Kammerer [13] describes an algorithm that can be used to construct a discriminant whose "shape" is prescribed. By the "shape" of a discriminant we are referring to the value of the discriminant at each of its real, distinct local extrema. For applications of Kammerer's algorithm we refer the reader to the forthcoming paper [5].

Useful information concerning properties of periodic Jacobi matrices is contained in [1], [9]. We would also like to state that the analysis presented in Section 3 can be extended in the same generality to "antiperiodic" Jacobi matrices of the form 


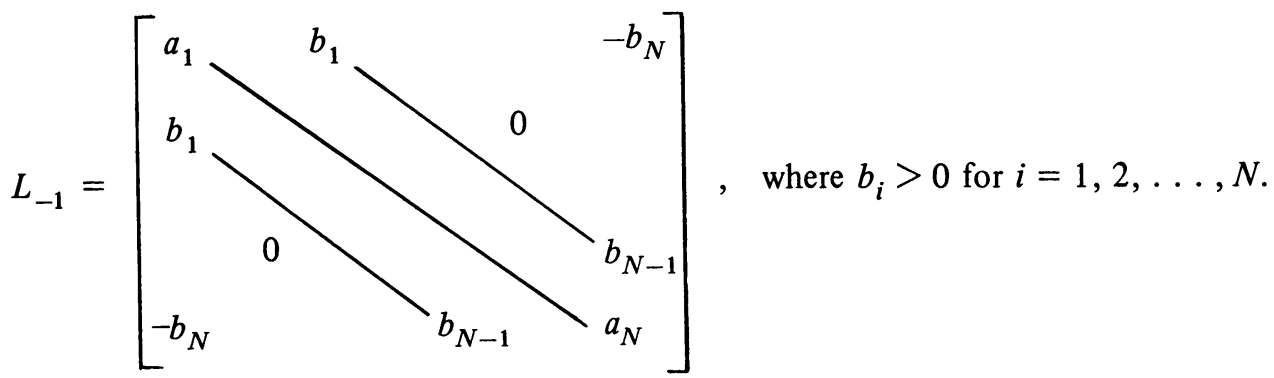

7. Acknowledgements. The author would like to thank C. de Boor, H. Flaschka, G. Golub and D. McLaughlin for several informative discussions. The author would also like to thank the agencies which support the Mathematics Research Center at the University of Wisconsin for allowing him to carry out this research.

Mathematics Research Center

University of Wisconsin-Madison

610 Walnut Street

Madison, Wisconsin 53706

Mathematics Department \#89

University of Arizona

Tucson, Arizona 85721

1. A. BJORCK \& G. GOLUB, "Eigenproblems for matrices associated with periodic boundary conditions," SIAM Rev., v. 19, 1977, pp. 5-16.

2. D. BOLEY \& G. GOLUB, Inverse Eigenvalue Problems for Band Matrices, Proc. Dundee Conf. on Numerical Analysis, Springer-Verlag, New York, 1977.

3. D. BOLEY \& G. GOLUB, The Matrix Inverse Eigenvalue Problem for Periodic Jacobi Matrices, STAN-CS-78-684, Computer Sciences Dept., Stanford Univ., Stanford, Calif., 1978.

4. C. DE BOOR \& G. GOLUB, The Numerically Stable Reconstruction of a Jacobi Matrix from Spectral Data, MRC-TSR-1727, Mathematics Research Center, Univ. of Wisconsin, Madison, Wis., 1977.

5. W. FERGUSON, H. FLASCHKA \& D. W. McLAUGHLIN, "Nonlinear normal modes for the periodic Toda lattice." (Preprint.)

6. H. FLASCHKA, "The Toda lattice," Phys. Rev. B, v. 9, 1974, pp. 1924-1925.

7. H. FLASCHKA \& D. W. McLAUGHLIN, "Canonically conjugate variables for the Korteweg-de Vries equation and the Toda lattice with periodic boundary conditions," Progr. Theoret. Phys., v. 55, 1976, pp. 438-456.

8. F. R. GANTMACHER \& M. G. KREIN, Oszillations-matrizen, oszillationskerne und kleine Schwingungen mechenischer Systeme, Akademie-Verlag, Berlin, 1960.

9. G. H. GOLUB, "Some modified matrix eigenvalue problems," SIAM Rev., v. 15, 1973, pp. $318-334$.

10. G. GOLUB \& J. WELSCH, "Calculations of Gauss quadrature rules," Math. Comp., v. 23, 1969, pp. 221-230.

11. O. HALD, "Inverse eigenvalue problems for Jacobi matrices," Linear Algebra and Appl., v. 14,1976, pp. $63-85$.

12. H. HOCHSTADT, "On the construction of a Jacobi matrix from spectral data," Linear Algebra and Appl., v. 8, 1974, pp. 435-446.

13. W. KAMMERER, "Polynomial approximations to finitely oscillating functions," Math. Comp., v. 15, 1961, pp. 115-119.

14. W. MAGNES \& S. WINKLER, Hill's Equation, Wiley, New York, 1965.

15. P. VAN MOERBEKE, "The spectrum of Jacobi matrices," Invent. Math., v. 37, 1976, pp. 45-81.

16. G. STEWART, Introduction to Matrix Computations, Academic Press, New York, 1973.

17. J. H. WILKINSON, The Algebraic Eigenvalue Problem, Oxford Univ. Press, London, 1965

18. J. H. WILKINSON \& C. REINSCH, Linear Algebra, Springer-Verlag, New York, 1971. 